\title{
Geriatric rehabilitation: program of care after orthopedic surgery for proximal femoral fractures
}

\begin{abstract}
Current opinion article underlines the importance of geriatric rehabilitation in clinical practice. We explain the potential of physical modalities for prevention of frequent problems in geriatric population: instability, falls, osteoporosis.

We present a detailed program of care of patients after proximal femoral fractures (diaphyseal or intertrochanteric), with subsequent orthopedic surgery (different interventions - hip replacement, re-arthroplasty, metallic osteosynthesis with plaque, intra medullary nailing, etc.). We emphasize on possible complications after these orthopedic interventions in geriatric patients with osteoporosis and postural instability (luxation of the head of the arthroplasty, luxation of the metallic osteosynthesis, formation of a false joint, peri-prosthetic fractures).

We consider that geriatric orthopedic rehabilitation can be very useful for the patients' autonomy and quality of life.

Keywords: geriatric rehabilitation, postural instability, imbalance, falls, osteoporosis, intertrochanteric fracture, hip replacement, periprosthetic fracture
\end{abstract}

\author{
Volume 5 Issue 5 - 2020 \\ Ivet Koleva, 1,2 Borislav Yoshinov, ${ }^{3}$ Julieta \\ Gerenova,' Radoslav R Yoshinov ${ }^{4}$ \\ 'Medical University of Sofia, Bulgaria \\ 'Long-term care and Rehabilitation hospital "Serdika", Bulgaria \\ ${ }^{3}$ Medical Faculty of Sofia University, Bulgaria \\ ${ }^{4}$ University of Telecommunications, Bulgaria

\begin{abstract}
Correspondence: Ivet Koleva, MD, PhD, DMedSc, Senior FEBPRM, Medical Doctor - Specialist in Physical and Rehabilitation Medicine (PRM),MD, Specialist in Neurology, Senior Fellow of the European Board of Physical and Rehabilitation Medicine, Philosophy Doctor in PRM, Philosophy Doctor in Pedagogics, Doctor of Medical Sciences in PRM, Tel +359888208I6I,

Email yvette@cc.bas.bg,dr.yvette.@gmail.com
\end{abstract}

Received: June 24, 2020 | Published: September 04, 2020

\section{Introduction}

\section{Motivation}

During last years, we observe the aging of the world population, respectively growth of the relative part of older people in the global structure of the population; ${ }^{2}$ this is the cause of the increased interest to the geriatrics and the geriatric care.

According the prognostics of United Nations ${ }^{3}$ the number of people over 60 years will duplicate at 2050. Older population is growing faster than the younger population. ${ }^{4,5}$ According the data of EUROSTAT the longevity is increasing. ${ }^{6}$

\section{Physical medicine, rehabilitation, ergo therapy}

According the Definition ${ }^{7}$ amended by the Council of the European Union of Medical Specialists (UEMS): "The medical act encompasses all the professional action, e.g. scientific, teaching, training and educational, clinical and medico-technical steps, performed to promote health and functioning, prevent diseases, provide diagnostic or therapeutic and rehabilitative care to patients, individuals, groups or communities in the framework of the respect of ethical and deontological value. It is the responsibility of, and must always be performed by a registered medical doctor / physician or under his or her direct supervision and/or prescription."

According the definitions ${ }^{8,9,10}$ in the White Book of the specialty Physical and Rehabilitation Medicine (PRM), formulated by the UEMS - PRM Section and Board: PRM is an "independent medical specialty, oriented to the promotion of physical and cognitive functioning, activities (including environment), participation (including quality of life) and changes in personal factors and environment. It is thus responsible for the prevention, diagnosis, treatments and rehabilitation management of people with disabling medical conditions and co-morbidity across all ages."

PRM is a "Medicine of Functioning", focusing on the improvement of functioning. Our work is based on the International Classification of Diseases ${ }^{11}$ and on the International Classification of Functioning, disability and Health ${ }^{12}[\mathrm{ICF}]$ (Figure 1).

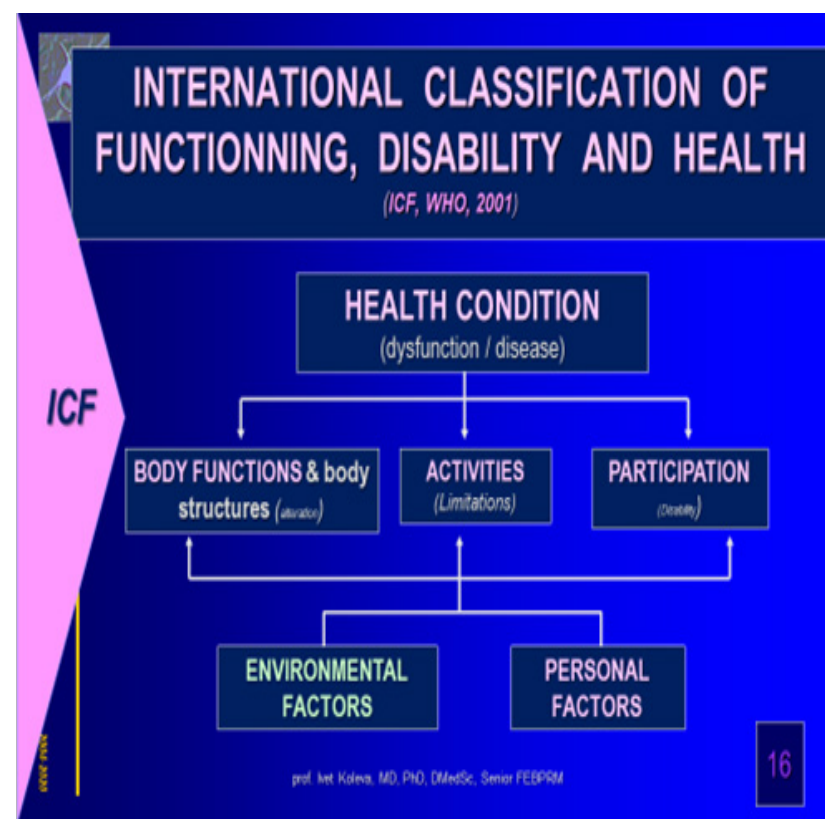

Figure I ICF. 
The diagnosis in PRM is the interaction between the medical diagnosis and a PRM-specific functional assessment. Outcomes of PRM interventions and programs, showed reduction of impairments in body functions, activity limitations, and impacting on participation restrictions, and also reduction in costs as well as decrease in mortality for certain groups of patients.

The goal of PRM is prevention, treatment and rehabilitation. In the clinical management of patients with diseases and conditions of the nervous, locomotor or cardio-vascular systems, the role of medical doctors - PRM specialists is significant.

Interventions in PRM are either provided directly by PRM physicians or within the rehabilitation team. They include a wide range of treatments, including medicaments, physiotherapy, ergotherapy, electrotherapy, education and many others. The principle of the rehabilitation 'puzzle' is presented in next Figure 2.

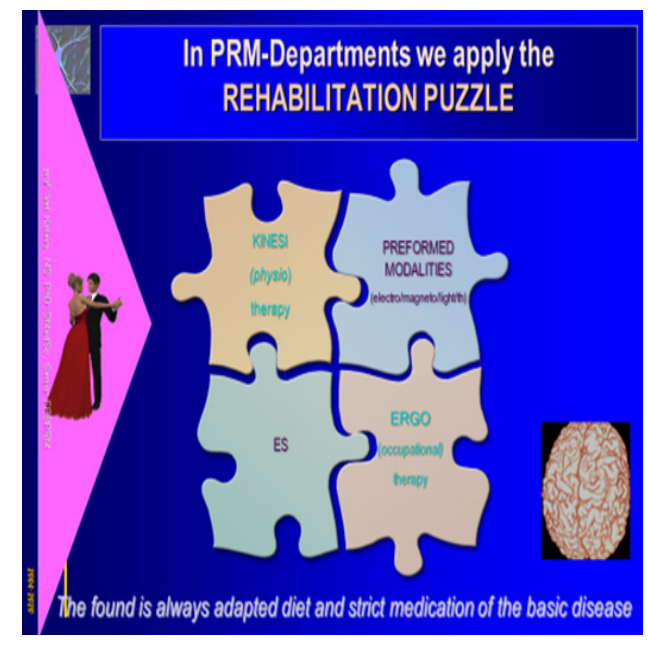

Figure 2 The "Rehabilitation Puzzle", applied in PRM departments.

The rehabilitation algorithm includes detailed functional evaluation (based on ICF) and synergic combination of physical modalities (movement, activities, mineral waters, electric currents, etc.). The "rehabilitation puzzle" (Figures 2 \& 3) includes many natural physical modalities [water (mineral baths), air, sun, exercises, massage, manual therapy techniques (traction, mobilization, and manipulation); ergotherapy (work and activities)] and pre-formed physical modalities [electric currents, light, magnetic field, ultra-sound, etc.].

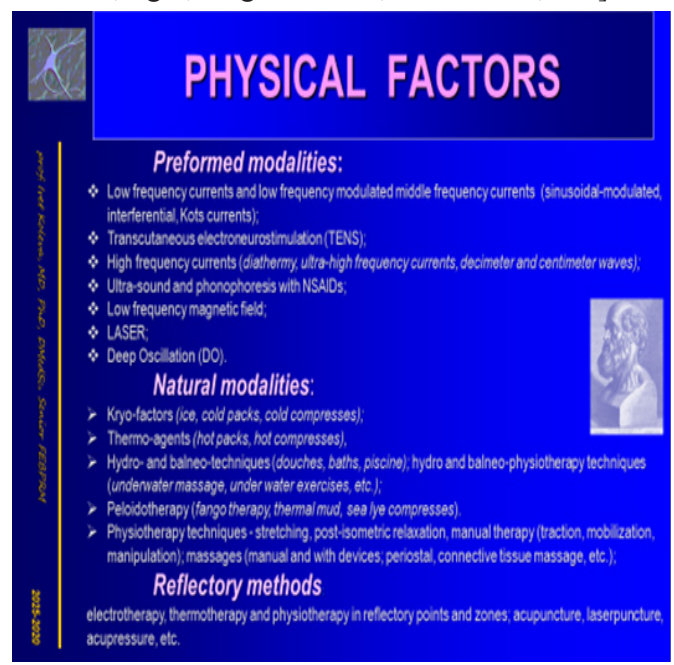

Figure 3 Physical modalities (physical factors).
The general rehabilitation algorithm includes one or two pre-formed modalities, one thermo- or cryo-agent, two or three physiotherapeutic procedures (including analytic exercises, post-isometric relaxation, stretching techniques, massage, etc.).

Rehabilitation is a functional therapy, realized in acute and chronic stage departments of hospitals for active or for long-term care; by a multi-disciplinary multi-professional team (medical doctors, nurses, physiotherapists, occupational therapists). The World Health Organization's (WHO) definition of rehabilitation is: "The use of all means aimed to reduce the impact of disabling and handicapping conditions and at enabling people with disabilities to achieve optimal social integration”. The World Report on Disability ${ }^{13}$ of the World Health Organization and World Bank defines the goals of rehabilitation: prevention and slowing the rate of loss of function; improvement, restoration or compensation of lost function; maintenance of current function.

Modern rehabilitation has an integrative and holistic approach to the patient, based on ICF and on clinical principles. The rehabilitation process according to the so-called rehabilitation cycle includes an assessment and definition of the (individual) rehabilitation goals, assignment to the rehabilitation program evaluation of individual outcomes.

The rehabilitation process is realized by a group of medical specialists and health professionals - the "Rehabilitation Team" (Figure 4).

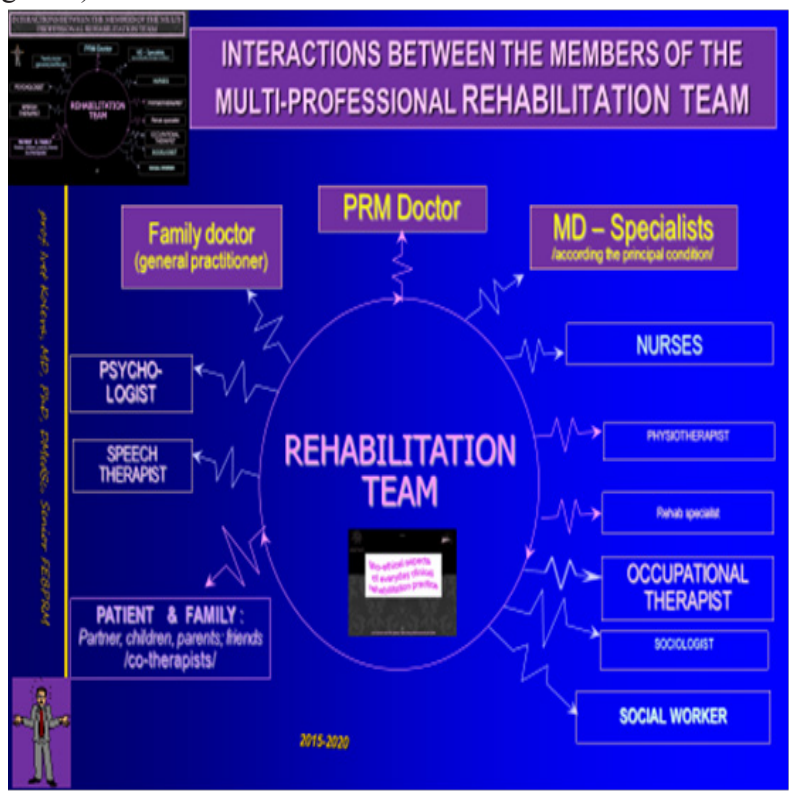

Figure 4 Members of the rehabilitation team.

The number of older chronic patients with invalidating diseases and conditions (predominantly of the nervous, motor and cardiovascular systems) increases during last years. All these persons have somato-sensory, motor and/or cognitive dysfunctions and deficits (Figure 5). Therefore, they need a complex rehabilitation programs, oriented to functional recovery and amelioration of their quality of life. In this process, the impact of PRM and rehabilitation is central.

Ergotherapy (ET) or Occupational therapy (OT) is part of the complex rehabilitation process. By definition, ${ }^{14}$ this is the use of purposeful activity with individuals, limited by physical injury or illness, psychosocial dysfunction, learning disabilities, poverty and cultural differences, or the aging process, with the goal to maximize 
independence, prevent disability, and maintain health.The ETpractice $^{15}$ encompasses evaluation, treatment and consultation. ETfields include: patients' education, adaptation of the environment to the requirements of patients with disability, and use of aiding devices (splints, belts, crutches, wheelchair, etc.).

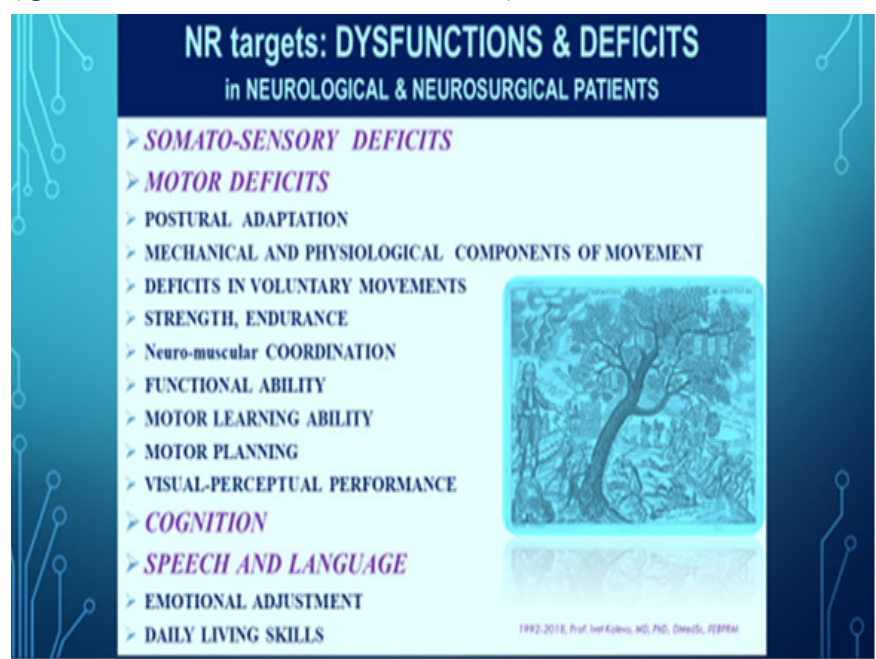

Figure 5 Frequent dysfunctions and deficits in geriatric rehabilitation clinical practice.

\section{Gerontology and geriatrics, Geriatric giants}

\section{Ortho-geriatrics and geriatric traumatology}

Geriatrics or geriatric medicine is considered as the science, learning the medical aspects of aging - normal and pathologic. Gerontology is the study of factors and mechanisms of the aging process.

The origin of the terms is from Greak: $\gamma \varepsilon$ $\rho \omega \nu$ (geron) = "old person”, $1 \alpha \tau \rho o ́ \varsigma($ iatros $)=$, ,therapist”.

The development of modern geriatrics in Europe is related to the name of Dr Marjorie Warren ${ }^{16}$ of London. She emphasized the polimorbidity of the older people, the role of the multi-disciplinary team and the efficacy of rehabilitation in the treatment of geriatric patients.

As father of modern geriatric medicine in England is considered Dr Bernard Isaacs,${ }^{17}$ who formulated the rule of the "geriatric giants" or the four I's: impairment of intellect (cerebral dysfunction), incontinence, immobility and instability (falls).Actually, the fifth letter I is added to the group of geriatric giants and the rule is called "the 5 I": Immobility, Instability, Incontinence, Impaired intellect/ memory, Impaired vision and hearing loss.

Ultimately, in the field of geriatrics, many medico-clinical and medico-surgical sub-specialties were developed. From the group of clinical sub-specialties we must empha size cardio-geriatrics, neurogeriatrics [cerebro-vascular diseases /as stroke/; neuro-degenerative conditions /as Alzheimer/; vascular or degenerative dementia]; onco-geriatrics; psycho-geriatrics (dementia, delirium, depression); geriatric rheumatology, geriatric rehabilitation. From the group of surgical geriatric sub-specialties the most important are:orthogeriatrics and geriatric traumatology (osteoporotic fractures, especially intertrochanteric), geriatric cardio-surgery.
Older people suffer from many diseases and conditions, ${ }^{18}$ falls, reduced autonomy, social isolation, ${ }^{19}$ depression, reduced quality of life. So, in the case of geriatrics, we can observe the Ockham's razor (the role of the age as Lex parsimoniae) and the Hickam's dictum (multiple conditions/polimorbidity). The holistic approach is obligatory. The necessity of care in rehabilitation departments is imposed.

"Geriatric giants" are the principal cause for falls and fractures of the lower limbs in older and frail persons, respectively, for the development of innovative surgical techniques in the field.

\section{Geriatric rehabilitation}

Geriatric rehabilitation ${ }^{20-24}$ includes three principal areas: normal aging, vascular problems (cardio-vascular disease and stroke) and musculo-skeletal problems (osteoporosis and osteoarthritis), and the subsequent treatments and complications (falls, fractures, hip and knee replacements). This is the cause of our interest on rehabilitation of the femoral fractures and the necessary PRM programs of care

Traditionally, geriatric rehabilitation ${ }^{24-28}$ comprises multidisciplinary care and physiotherapy. Authors emphasize on the frequency of fragility fractures ${ }^{25}$ on specific surgical and anesthesia techniques, ${ }^{26}$ on the organization ${ }^{27,28}$ of the post-operative care.

\section{Goal of current article}

We consider that in geriatric cases the inclusion of other physical modalities is necessary and can be useful for our patients.

So, the Goal of current article is to suggest a complex rehabilitation program in cases after orthopedic surgery for proximal femoral fractures: intertrochanteric or per-trochanteric fractures, fractures of the proximal diaphysis, periprosthetic fractures.

\section{Geriatric rehabilitation program after orthopedic surgery for proximal femoral fractures}

\section{Type of patients for geriatric ortho-rehabilitation}

Patients after orthopaedic surgery for proximal femoral fractures are transferred from the orthopaedic surgery department to the PRMdepartment at an ever earlier stage.

In geriatric rehabilitation, major goals are the improvement of the range of motion (ROM) of the hip, verticalization of the patient and gait training with gradual Weight-Bearing (WB - from $0 \%$ to $100 \%$ ). Next figures (Figures 6-12) present data of some of our in-patients (X-ray) at the admission in the orthopaedic department (Figure 6) and / or in our PRM-Department (Figures 7-12). We proceed the rehabilitation of different type of patients: with Intertrochanteric fractures and subsequent hip replacement (Figure 6,7), with metallic osteosynthesis of the femoral body after a peri-prosthetic fracture (previous hip replacement) (Figure 8), with hip re-arthroplasty after a periprostheticdiaphyseal femoral fracture (Figure 9), with inter-trochanteric fracture and subsequent orthopedic surgery intramedullary nailing (Figure10), with per-trochanteric fracture, operated with implantation of metallic osteosynthesis - PCOM with DHS plaque (Figure 11), with proximal diaphyseal femoral fracture with dislocation and intervention of PCOM with DHS plaque (Figure 12). 


\section{Intertrochanteric fractures, with subsequent hip replacement}

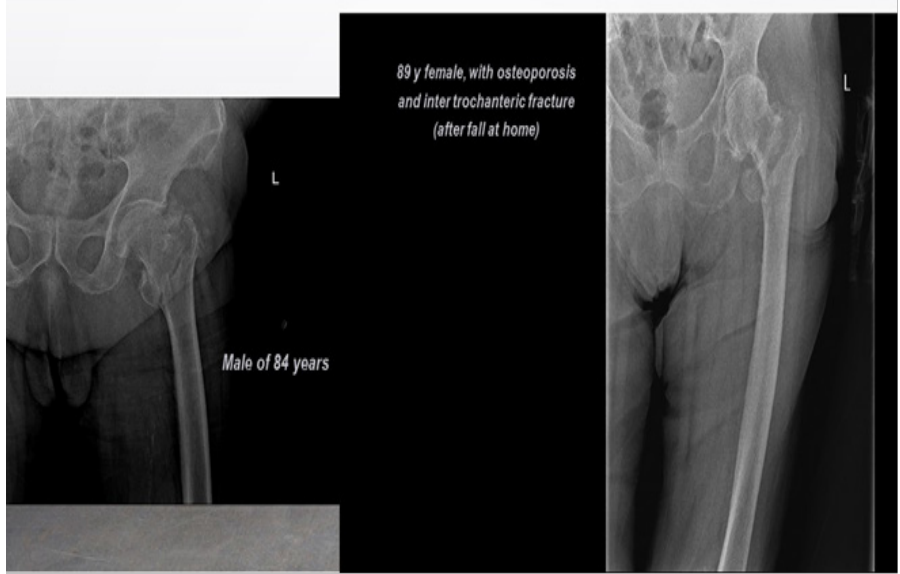

Figure 6 Intertrochanteric fractures, future hip replacement.

68 years old man, with operation of the left hip - hip arthroplasty after per trochanteric fracture

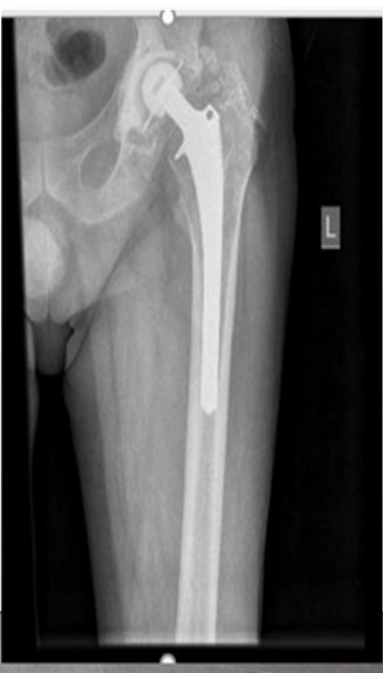

Figure 7 Hip replacement after per-trochanteric fracture.

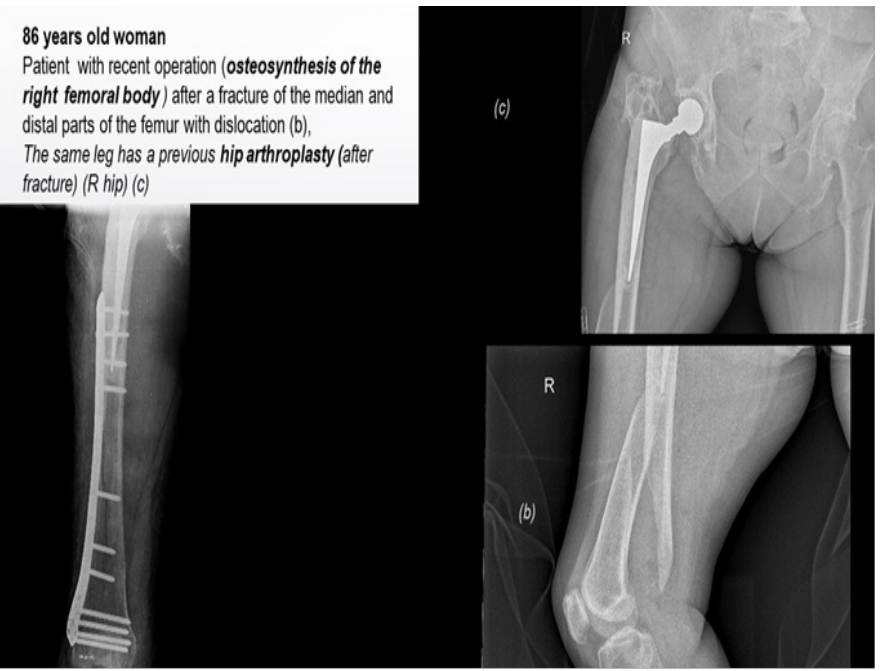

Figure 8 Metallic osteo-synthesis of the right femoral body after a periprosthetic fracture (previous hip replacement).

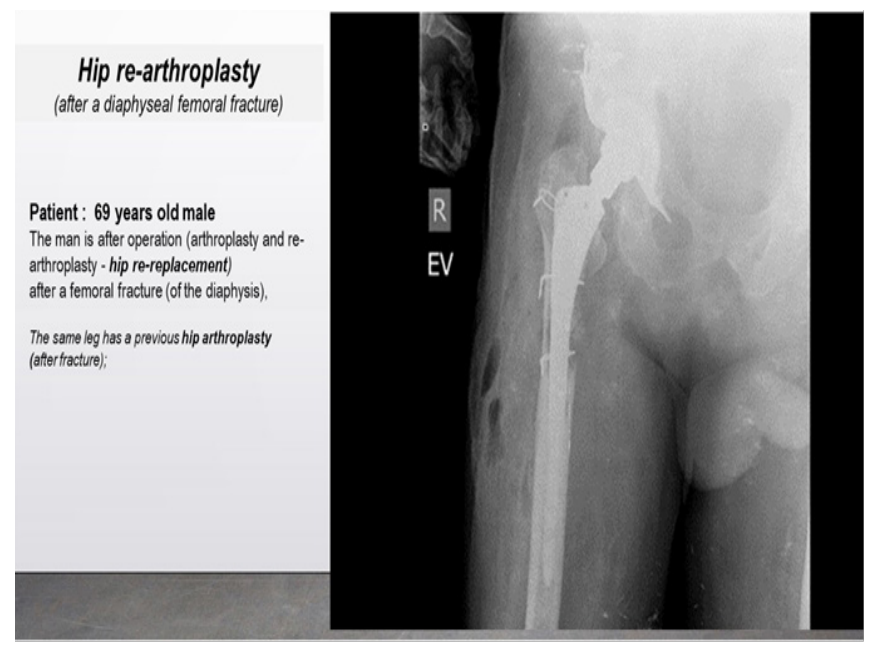

Figure 9 Hip re-arthroplasty after a periprosthetic diaphyseal femoral fracture.

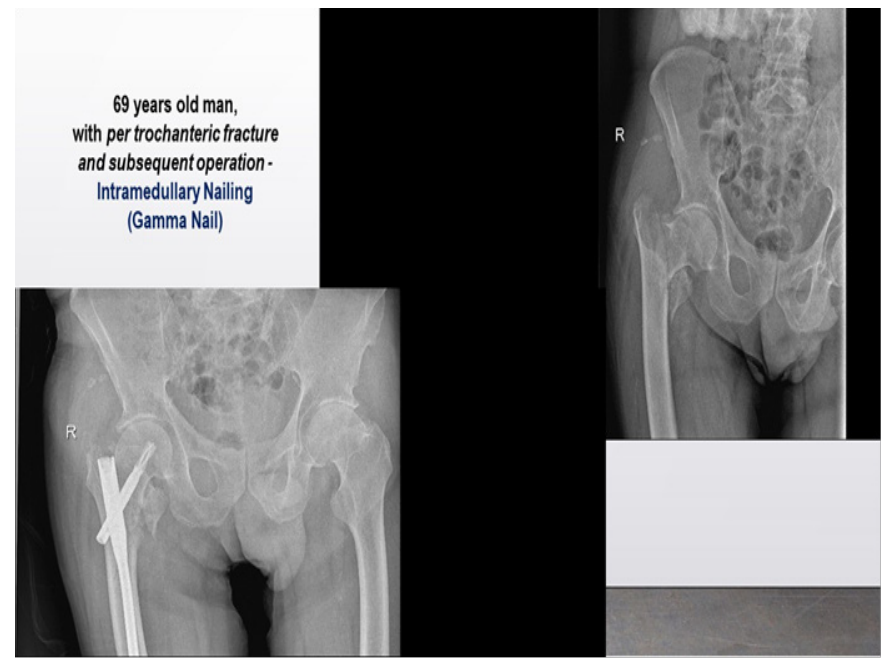

Figure 10 Patient with inter-trochanteric fracture and subsequent orthopedic surgery - intramedullary nailing.

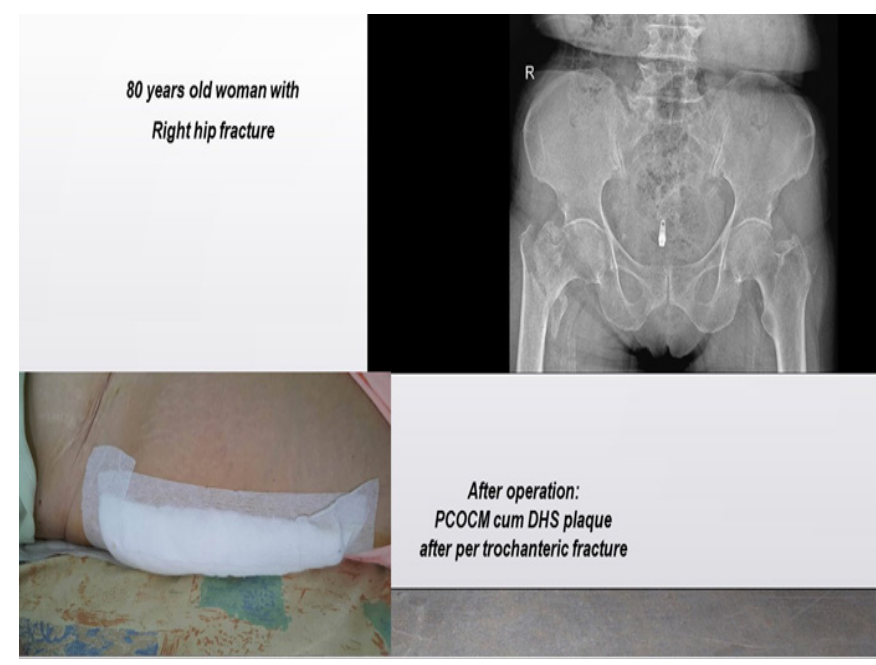

Figure II X-ray of a per-trochanteric fracture - and the patient after the orthopedic surgery (metallic osteosynthesis - PCOM with DHS plaque). 


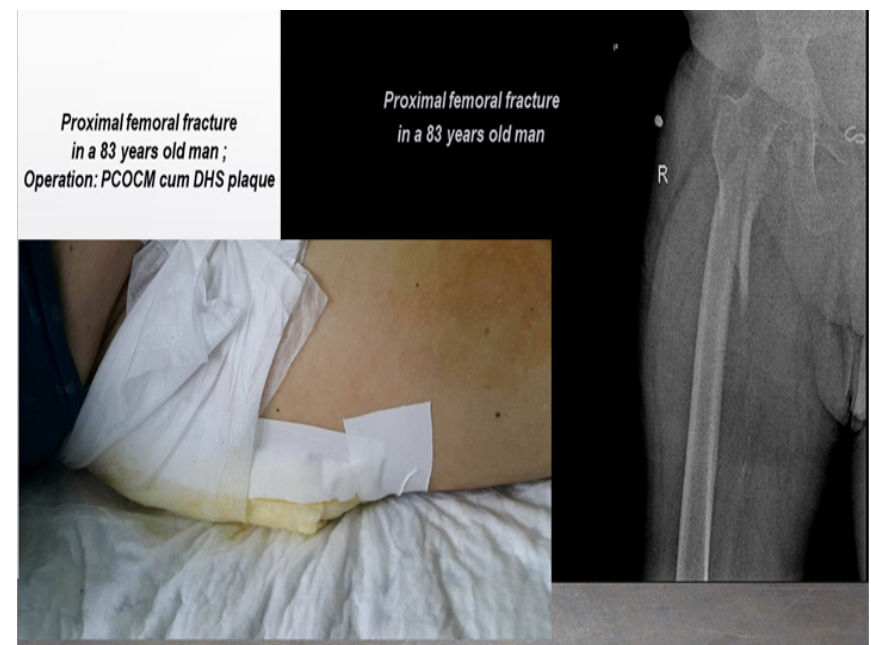

Figure 12 X-ray of a proximal diaphyseal femoral fracture with dislocation and patient after intervention (PCOM with DHS plaque).

\section{Goals of rehabilitation in ortho-geriatric clinical practice} follows:

The principal training goals in the geriatric rehabilitation are, as

1. Improvement of motor skills, cardiovascular conditions and strength;

2. Improvement of circulation and prevention of muscular hypotrophy;

3. Prevention of contractures;

4. Prevention of deep vein thrombosis in lower limbs;

5. Rapid muscle build-up: analytic exercises for quadriceps and gluteal muscles, mechano-therapy;

6. Early start of active training; for older patients - combination of passive-assistive and active training;

7. Active cycling against a minimal resistance (we increase resistance progressively);

8. Restoration of the correct gait pattern.

The applied physiotherapeutic methods and devices of the PRMDepartment (Figure 13).

\section{Rehabilitation program in a patient with proximal femoral fracture after orthopedic surgery (total hip arthroplasty, metallic osteo synthesis or intra medullary nail)}

Patient Complaints at the admission in the PRM-Department are: Excessive pain and stiffness in the fractured hip or femur and muscles around it , Difficulties in standing up, transfers and mobility, Reduction of autonomy in activities of daily living (ADL); Gait with walker or crutches; with or without assistant.

The Clinical exam demonstrates: Limited range of motion of the correspondent lower extremity. We survey the post-operative cicatrix (for eventual complications).

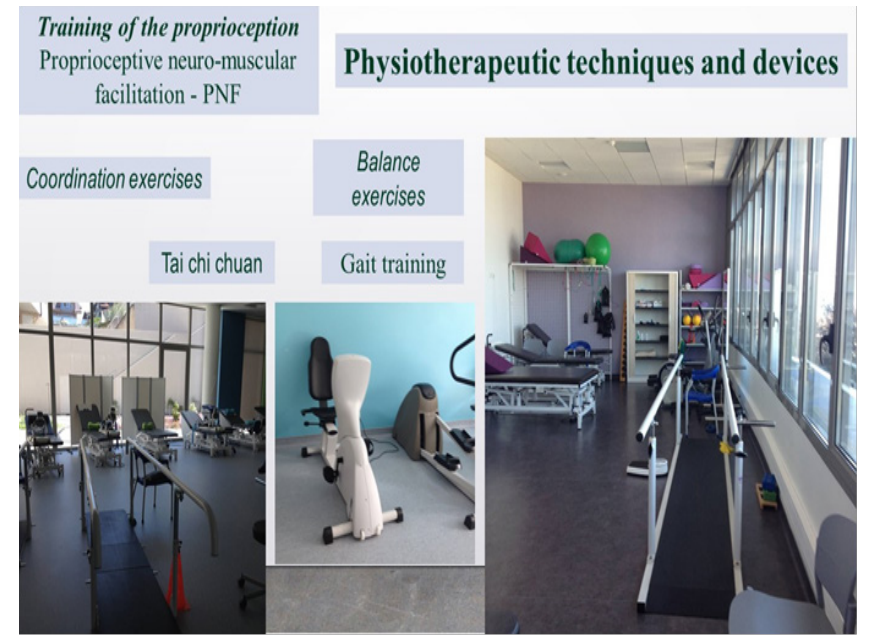

Figure I 3 Physiotherapeutic methods and devices.

\section{The functional assessment includes kinesiological analysis and a detailed ICF-assessment}

Kinesiological analysis usually demonstrates: limited range of motion (ROM evaluated by goniometry of the operated hip joint in flexion and abduction; flexion over $90^{\circ}$ and adduction are strictly interdicted); difficulty in transfers, impossible normal gait; the patient can or can't effectuate 10 meters walk test - with walker, one or two crutches and an assistant PT. We evaluate the rehabilitation potential and the co-morbidities.

The ICF assessment validates: impairments of body functions (hip pain, muscle weakness, and restricted hip ROM);changes in body structures; activity limitation (limited walking ability and problems with putting on socks); participation restrictions (reduced participation in everyday and leisure activities); decrease of the patient's level of autonomy.

We realize some evaluation tests:

10 meters walk test $(10 \mathrm{mWT})$ - seconds before \& after rehab;

Timed Up and Go test (TUG) - seconds after rehabilitation;

Minutes Walk Test (6 MWT)- impossible before rehabilitation; xxx meters after rehabilitation (after the inpatient rehab cycle).

\section{Rehabilitation program}

The GOAL is functional recovery and reeducation Principal TASKS are: recovery of the stability and mobility of the lower limb joints; restoration of the muscle and ligament balance, accentuating on muscles around the hip joint; keeping the hip in the economic limb biomechanics; pain control; control of the cicatrix; control of joint ROM; control of possible complications; verticalization and education of transfers; normal gait recovery with correction of eventual abnormal walking scheme; ADL (activities of daily living) training; amelioration of autonomy in everyday life; psycho-emotional stimulation; amelioration of the health-related quality of life.

We use diverse METHODS:

i. Drugs (Fraxiparine; analgesics);

ii. Patient's education;

iii. Posture (activity modification); 
iv. Electrotherapy - magnetic field, interferential currents;

v. Cryotherapy - for the hip joint (before the massage and the physiotherapy);

vi. Massage - classic massage (relaxing for the anterior group of muscles of the hip; stimulating for gluteal muscles);

vii. Individualized physiotherapeutic program - correct posture of lower limb, range-of-motion (ROM) exercises for joints of lower extremities (Figure 14); analytic exercises for gluteal muscles especially for gluteus medius muscle; lower limb joint mobilization (active range of motion) ;post-isometric relaxation /PIR/ for iliopsoas muscle;

viii. Gait training with supporting walker, rollator or two crutches (Figure 15-16), education in mobility - up and down the stairs;

ix. Occupational therapy \& training in activities of daily living (ADL) (Figure 17).

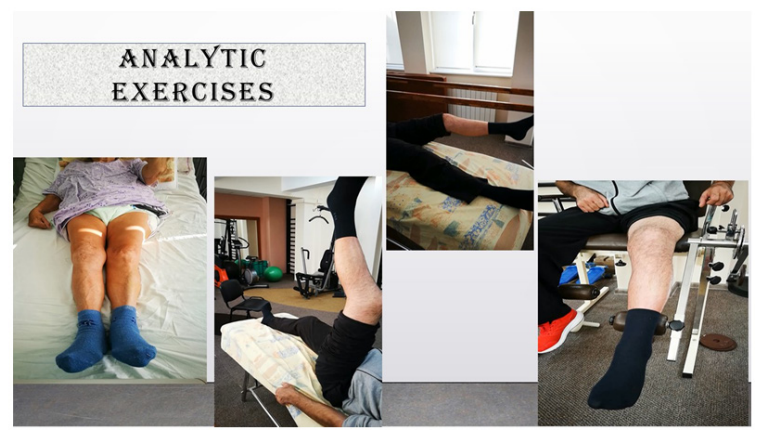

Figure I4 ROM and analytic exercises of the lower limbs: for ankles, knees and hips.

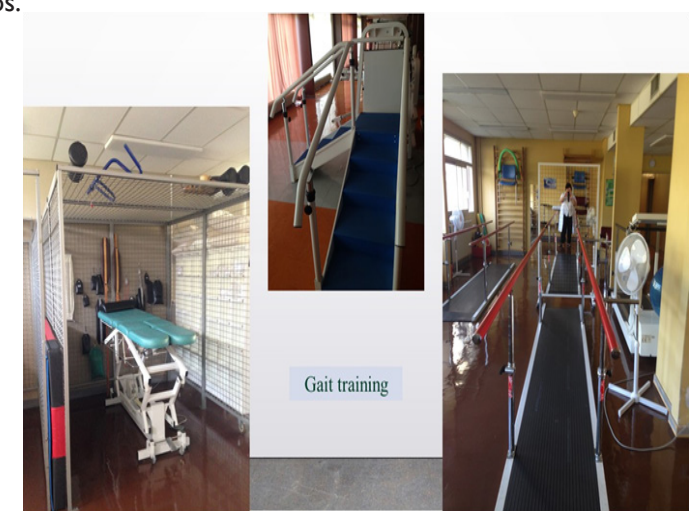

Figure 15 Devices for gait training.

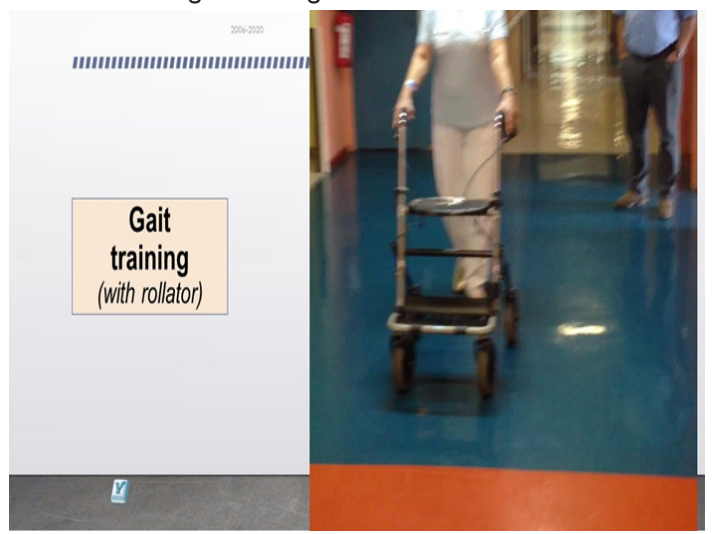

Figure 16 Gait training with rollator.

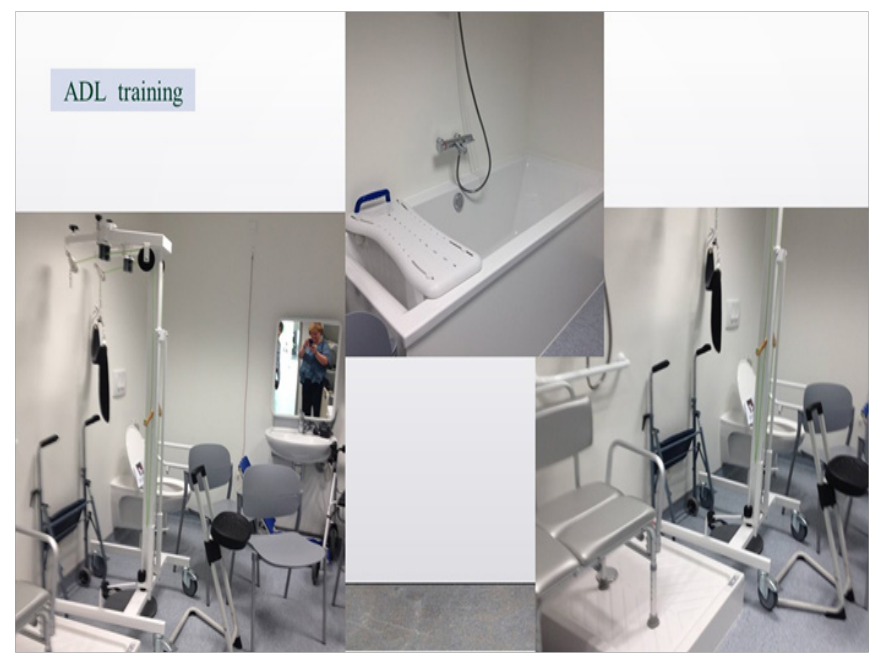

Figure 17 Training in activities of daily life (ADL).

The functional assessment at the end of the inpatient rehabilitation includes Goniometry of the operated hip and 6 Minutes Walk Test (6-MWT) - in meters

We obtain positive RESULTS of the applied PRM-program: Amelioration of the range of motion of the corresponding hip;Amelioration of the functional capacity;Independent stand up and transfers; Independent gait with crutches - in the room and the corridor; Balance \& Gait stabilization; Decrease of dysesthesias and pain in distal parts of lower limbs;Amelioration of the autonomy in ADL.

We formulate some RECOMMENDATIONS after discharge: Auto-PT at home (physiotherapy every day at the thirdmonth after the operation); Gait - with walker, one or two crutches - with controlled weight-bearing; the next rehabilitation course at hospital is usually planned 3 months after orthopedic surgery.

The general Hip-conditioning program for long-life protection after surgery is oriented to stimulate the patient to return to daily activities, to sport and other recreational activities. We recommend Strengthening exercises and Flexibility exercises, with stretching the muscles for restoration of ROM and prevention of future injuries. The Target musclesare: Gluteus maximus, Gluteus medius; Hamstrings; Piriformis; Hip adductors and hip abductors; Tensor fasciae latae. All exercises must be done without pain (or increase of current pain). Patient can realize exercises at home, only if the therapist is sure that the application of exercises will be correct!.

\section{Complications after lower limbs orthopedic surgery in geriatric patients}

Next figures (Figure 18-20) present some complications, due to osteoporosis, successive falls or inappropriate rehabilitation luxation of the head of the arthroplasty (Figure 18), luxation of the osteosynthesis with formation of a "false" hip joint (Figure 19), proximal or distal femoral fracture after hip replacement (Figure 20).

\section{Conclusion}

The suggested complex PRM-program is an example. We consider that different elements of the program must be adapted to the individual patient, in the concrete stage of his post-operative rehabilitation. 


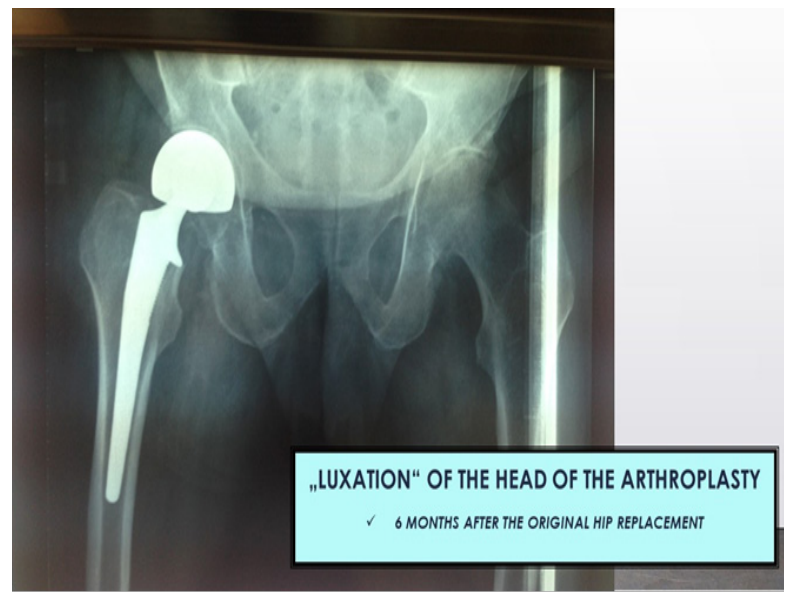

Figure 18 Luxation of the head of the arthroplasty.

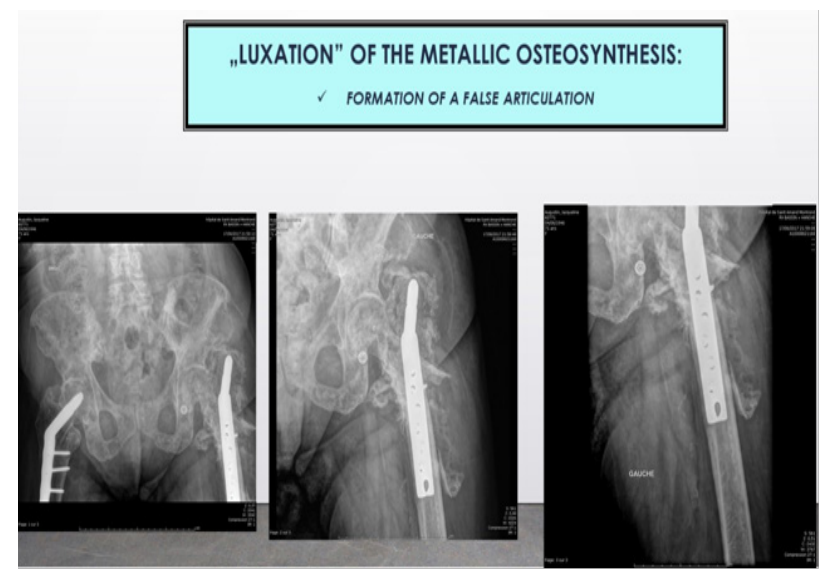

Figure 19 Luxation of the metallic osteosynthesis with development of a false joint.

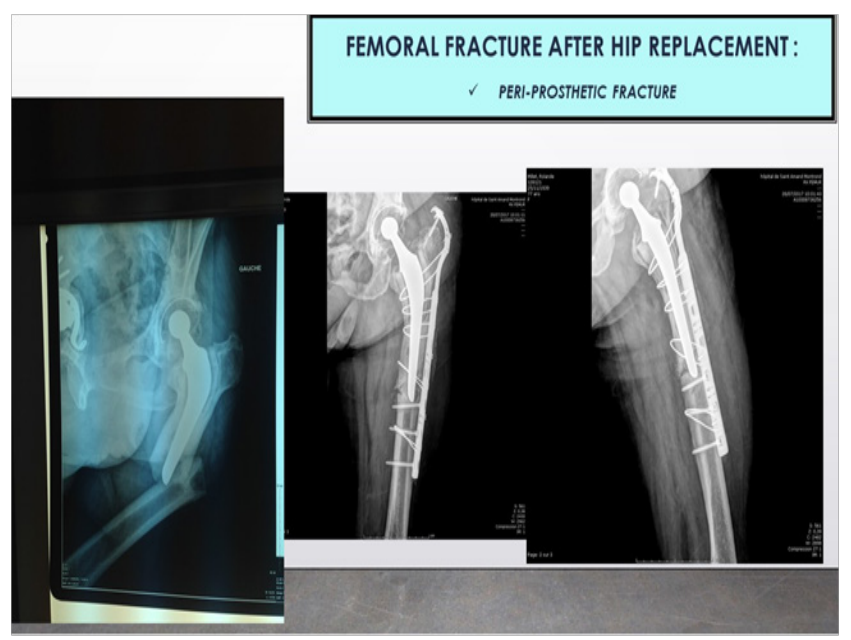

Figure 20 Periprosthetic femoral fracture with dislocation after fall at home of a patient with arthroplasty.

Our modest clinical practice of $30+$ years in the field ${ }^{29}$ proved that the in-time competent rehabilitation is the key element in the combat against the geriatric giant instability, with the subsequent falls, osteoporotic fractures, orthopedic surgery and rehabilitation. We consider that geriatric rehabilitation have the potential to increase the quality of life of our patients. ${ }^{29}$

\section{Funding information}

Authors declare that the current article was prepared without sources of funding.

\section{Acknowledgments}

No.

\section{Competing interests}

Authors have declared that no competing interests exist.

\section{References}

1. World report on ageing and health by World Health Organization. 2015.

2. United nations department of economic and social affairs population division. World population is ageing 2013.

3. Department of Social and Economic Affairs, Population Division. World population is ageing 1950-2050, United Nations; 2001.

4. Federal interagency forum on aging related statistics. Older Americans 2000: Key indicators of wellbeing. Washington DC: U.S. government printing office; 2000.

5. Kinsella K, Wan H. U.S. Census Bureau, International Population Reports, p95/09-1, An Aging World: 2008, U.S. Government Printing Office, Washington, DC, 2009.

6. Centers for disease control and prevention (CDC). Trends in aging: united states and worldwide. MMWR Morb Mortal Wkly Rep. 2003;52(6):101104.

7. UEMS (Union européenne des médecins spécialistes) 2005/14 final. European definition of the medical act. adopted in munich,.2005.

8. UEMS - PRM-section: Definition of physical and rehabilitation medicine. 2005 .

9. White book on physical and rehabilitation medicine in europe. Produced by the section of physical and rehabilitation medicine, Union Européenne des Médecins Spécialistes (UEMS), the european board of physical and rehabilitation medicine and l'académie européenne de medicine de réadaptation in conjunction with the european society of physical and rehabilitation medicine (ESPRM). C Gutenbrunner, $\mathrm{AB}$ Ward, MA Chamberlain Editors. Journal of Rehabilitation Medicine, 2007;1(45):148.

10. White book on physical and rehabilitation medicine (PRM) in Europe. Chapter 7. The clinical field of competence: PRM in practice. European Physical and Rehabilitation Medicine Bodies Alliance. Eur J Phys Rehabil Med. 2018;54(2):230-260.

11. World health organization. The international statistical classification of diseases and health-related problems ICD -10. Second Edition. - Geneva: WHO, 2005.

12. World health organization. International classification of functioning, disability and health (ICF). - WHO, Geneva, 2001

13. World health organization. World report on disability. May 15, 2012.

14. AK Evans. Nationally speaking: definition of occupational therapy. AJOT. 1987;(41)10:627-628.

15. Resolution Q: Definition of occupational therapy for licensure. Minutes of the 1981 AOTA representative Assembly. AJOT. 1981;35:798-799.

16. Denham MJ. Marjory warren CBE MRCS LRCP (1897-1960) the mother of British geriatric medicine. J Med Biogr. 2011;19(3):105-110.

17. A giant of geriatric medicine - Professor Bernard Isaacs (1924-1995)". British Geriatrics Society. 2019. 
18. Hughes LD, McMurdo MET, Guthrie B. Guidelines for people not for diseases: the challenges of applying UK clinical guidelines to older people with multiple co-morbidities. Age Ageing. 2013;42(1):62-69.

19. World health organization. social isolation and loneliness in older adults: opportunities for the health care system. Geriatry Consensus. Consensus Study Report. 2020.

20. Wedgwood J. The place of rehabilitation in geriatric medicine; an overview. Int Rehabil Med. 1985;7(3):107-109.

21. Grabbe JW. Recent advances in geriatric medicine (An interdisciplinary approach to Geriatric medicine). Plattsburgh: Bentham Science Publishers, 2017;2:162.

22. Weber DC, Fleming KC, Evans JM. Rehabilitation of geriatric patients. Mayo Clin Proc. 1995;70(12):1198-1204.

23. Berk B, Cifu D. Geriatric rehabilitation - from bedside to curbside. Boca Raton: Taylor and Francis Group. 2017;713.
24. Rao Poduri K. Geriatric rehabilitation (from bedside to curbside). - CRC Press, Taylor \& Francis Group. 2017, 713.

25. British orthopaedic association and british geriatric society. The care of patients with fragility fracture (Blue Book); British Orthopaedic Association: East Sussex, UK, 2007.

26. White SM, Griffiths R. Projected incidence of proximal femoral fractures in England: A report from the NHS Hip Fracture Anaesthesia Network (HIPFAN). Injury. 2011; 42:1230-1233.

27. Middleton M. Ortho geriatrics and hip fracture care in the UK: factors driving change to more integrated models of care. Geriatrics. 2018;3:55.

28. Middleton M, Wan B. Da Assunção, R. Improving hip fracture outcomes with integrated orthogeriatric care: A comparison between two accepted orthogeriatric models. Age Ageing. 2017;43:465-470.

29. Koleva I. Orthopedic rehabilitation. Electronic book - Sofia, SIMEL press. $2020 ; 498$ 